\title{
Combinatorial effect of epigallocatechin-3-gallate and TRAIL on pancreatic cancer cell death
}

\author{
ARUNA BASU and SUBRATA HALDAR

\begin{abstract}
Center for Biomedical Sciences, Department of Pharmacology, Case Comprehensive Cancer Center,
\end{abstract} \\ MetroHealth Campus, Case Western Reserve University, Cleveland, OH, USA
}

Received July 11, 2008; Accepted September 10, 2008

DOI: 10.3892/ijo_00000150

\begin{abstract}
Epigallocatechin-3-gallate (EGCG), a major polyphenolic constituent of green tea, can exert growth suppressive effect on human pancreatic cancer cells by evoking apoptotic response. EGCG-induced apoptosis of pancreatic cancer cells is accompanied by growth arrest at an earlier phase of cell cycle along with depolarization of mitochondrial membrane. In this report, using MIA PaCa-2 cells as in vitro model, we demonstrate EGCG-induced cell death involves activation of caspase- 8 and disappearance of intact $21 \mathrm{kDa}$ Bid protein. Furthermore, exogenous expression of dominant negative caspase- 8 or dominant negative FADD significantly abrogates apoptosis inducing ability of EGCG in MIA PaCa-2 cells. RNase protection assay revealed upregulation of the members of death receptor family, thus indicating the involvement of transmembrane extrinsic signaling in this polyphenol triggered pancreatic carcinoma cell death. Based on this, we examined the effect of EGCG and tumor necrosis factor-related apoptosis-inducing ligand (TRAIL) together on pancreatic cancer cells. A synergistic increase in apoptosis and cleavage of procaspase-3 was noted. Furthermore, clonogenic cell survival assay demonstrates the significant diminishment of MIA PaCa-2 cell proliferation in the presence of both EGCG and TRAIL. This combination treatment strategy has potential therapeutic advantage for pancreatic carcinoma.
\end{abstract}

\section{Introduction}

The development of malignancies is a multi-step process that comprise of initiation, progression, invasion and finally establishment of metastasis. The multiple genetic alterations at each stage impart selective advantage over normal counterpart. In this respect, pancreatic carcinoma is no exception. During

Correspondence to: Dr Aruna Basu, R455, Rammelkamp Building, MetroHealth Medical Center, 2500 MetroHealth Drive, Cleveland, OH 44109, USA

E-mail: abasu@metrohealth.org

Key words: pancreatic cancer, apoptosis, EGCG, TRAIL, death receptor last decade, several cancer-causing genes have been found to be associated with pancreatic carcinoma (1-3). Undoubtedly, the identification of these cancer-related genes have substantially improved our knowledge on pancreatic cancer development. However, the treatment of this cancer has not advanced that much. This might be due to the lack of early diagnosis and effective chemotherapeutic treatments. In pursuit to achieve a greater survival rate of pancreatic cancer patients, new molecular targets for drug discovery, better diagnosis markers as well as development of potential chemopreventive agents are absolutely necessary at this stage.

Cancer prevention by employing naturally occurring agents against defined molecular targets is a rational approach (4-14). Catechins are the key components of tea that exert antiproliferative properties. Among them, the most abundant in tea extract, epigallocatechin-3-gallate (EGCG) was observed to effectively inhibit tumorigenesis and tumor growth in preclinical animal models $(15,16)$. The bioavailability studies with this compound reveal the evidence of its wide distribution in mouse organs including pancreas. Ample animal studies exhibited the broad spectrum cancer preventive activities of tea constituents in different organs (5). The mechanistic studies on the action of EGCG in different cancer cells indicate that multiple pathways might be involved to exert its anticancer activity. The ability of EGCG to inhibit MAP kinases and P13K/AKT pathway, NF-KB and AP-1 mediated transcription or growth factor mediated signaling was implicated with its cancer preventive action (5). Moreover, several reports documented its inhibitory effect on angiogenesis as shown by suppression of endothelial cell growth and production of proangiogenic factor, VEGF $(17,18)$.

Previously, we have observed that EGCG could invoke apoptosis in pancreatic carcinoma cells by Bax oligomerization, depolarization of mitochondrial membranes to facilitate cytochrome c release into cytosol (4). EGCG-induced stress signals also involved ROS mediated JNK activation in pancreatic cancer cells. The antiproliferative action of EGCG was associated with nuclear condensation, Poly-ADP ribose polymerase (PARP) cleavage as well as activation of executioner caspase-3.

Here we were interested to investigate the role of other caspases in EGCG mediated apoptosis. Our investigation indicates that EGCG mediated apoptosis of pancreatic carcinoma cells requires activation of both initiator and executioner caspases. In order to comprehend the mechanism of caspase- 8 activation we employed RNase protection assay 
(RPA) using multi-probe template (6) set. It is evident that EGCG exposure induces the upregulation of death receptors in pancreatic cancer.

Since EGCG is able to trigger death receptor signaling, we examined the effects of EGCG and death receptor ligand TRAIL together in cell culture model. Tumor necrosis factor (TNF)-related apoptosis inducing ligand (TRAIL) is a type II transmembrane cytokine and a potent inducer of apoptosis in cancer cells (6). Interestingly, polyphenol EGCG can cooperate with TRAIL to enhance the sensitivity of pancreatic cancer cells to apoptosis in vitro.

\section{Materials and methods}

Cell culture. Human pancreatic cancer cell lines MIA PaCa-2 and Hs 766T were obtained from the American Type Culture Collection (ATCC) (Manassas, VA, USA). Cells were grown in RPMI supplemented with $10 \%$ FBS and $50 \mu \mathrm{g} / \mathrm{ml}$ gentamicin at $37^{\circ} \mathrm{C}$ in a $5 \% \mathrm{CO}_{2}$ humidified atmosphere.

Apoptosis assay. Cells $\left(5 \times 10^{6}\right)$ were seeded (in triplicate plates) in the growth medium and the next day treated with EGCG (Sigma, MO) in the presence or absence of death receptor ligand TRAIL (R\&D Systems, MN) for designated time periods. Dominant negative cDNA constructs encoding caspase-8, -9, FADD (6) in mammalian expression vectors were transiently transfected $(6,19,20)$ prior to EGCG exposure. Subsequently, cells were either processed for chromatin condensation analysis by DNA group binding dye, 4', 6'diamidino -2-phenylindole (DAPI) fluorescence or poly-ADP ribose polymerase (PARP) cleavage analysis (21-25) by immunoblotting with monoclonal antibody against PARP (BD Biosciences, CA). For DAPI staining, cells were washed and fixed followed by mounting in a fluid containing $2 \mu \mathrm{g} / \mathrm{ml}$ DAPI (Vector Laboratories, CA). A Nikon Eclipse E600 Fluorescence microscope was used to visualize nuclear stain.

Immunoblotting. MIA PaCa-2 cells at 5x106/100 mm dish were seeded and treated 1 day later with specified concentrations of EGCG. For studies with death receptor ligands, cells were co-incubated with $5 \mathrm{ng} / \mathrm{ml}$ TRAIL (R\&D Systems, MN) and $0.1 \mathrm{mM}$ EGCG for designated time periods. Following treatment, total cellular proteins were extracted $(6-8,19-25)$. After normalization for total protein content, the resulting lysate was subjected to SDS-PAGE and blotted into nitrocellulose membranes (Amersham Biosciences, NJ). Membranes were probed with the following antibodies: procaspase-8, -9 (Santa Cruz Biotechnology, CA), procaspase-3 (BD Biosciences), Bid (Cell Signaling, MA), Survivin (Novus Biologicals, CO), PARP (BD Biosciences). Immunodetection was accomplished by enhanced chemiluminescence method (Amersham). Immunoblotting with $\alpha$-tubulin or $\beta$-actin antibodies (Sigma) was performed for protein loading control.

RNase protection assays. RNase protection assay (6) in control and EGCG treated pancreatic cancer cells was performed as described under the BD Riboquant multi-probe kit Instruction Manual (BD Biosciences). Total RNA (10 mg) isolated from each sample was hybridized with $8 \times 10^{5} \mathrm{cpm}$ of labeled probe template set hAPO-3d in hybridization buffer. Hybridization reactions were initiated at $90^{\circ} \mathrm{C}$ and allowed to cool down slowly to $56^{\circ} \mathrm{C}$. Subsequently, the samples were treated with RNaseA/RNaseT1 for $45 \mathrm{~min}$ at $30^{\circ} \mathrm{C}$ followed by proteinase $\mathrm{K}$ digestion for $15 \mathrm{~min}$ at $37^{\circ} \mathrm{C}$. The samples were purified by phenol/chloroform extraction and ethanol precipitation. Finally the samples were resuspended in loading buffer $(95 \%$ formamide; $0.025 \%$ xylene cyanol; $0.025 \%$ bromophenol blue; $0.5 \mathrm{mM}$ EDTA and $0.025 \%$ SDS). The RNase-protected fragments were analyzed on a $5 \%$ denaturing polyacrylamide gel (19:1 acrylamide/bis) followed by autoradiography. The intensity of the signals was quantitated using a PhosphorImager system (Molecular Dynamics, Storm 860) and normalized using the housekeeping gene L32.

Clonogenic cell survival assay. MIA PaCa- 2 cells were treated with EGCG alone, TRAIL alone or combination of EGCG and TRAIL for $24 \mathrm{~h}$. Following treatment, cells were washed two times with phosphate buffered saline and then seeded in triplicate $(10000$ cells $/ 10 \mathrm{~cm}$ dish). Cells were allowed to grow for additional two weeks in the absence of EGCG or TRAIL. Medium was changed every four days. Subsequently, cells were fixed with $4 \%$ buffered formalin and stained with $0.05 \%$ crystal violet for visualization and photography. The number of colonies was counted manually (26).

\section{Results}

EGCG mediated apoptosis requires activation of caspase-8 and -9 . Our previous studies (4) revealed that EGCG is capable of promoting apoptosis in pancreatic cancer cells by inducing mitochondrial membrane depolarization, the downregulation of IAP family member, XIAP as well as caspase-3 activation. In order to further understand the molecular mechanism of EGCG action on apoptosis of pancreatic cancer cells, we were interested to examine the involvement of other caspases such as caspase- 8 or -9 . For this purpose, MIA PaCa-2 cells were treated with EGCG for 8-24-h time period and the activity was assessed by the disappearance of the inactive forms of procaspase- 8 and -9 on Western blotting (Fig. 1). The two species of procaspases observed here conform to previous reports (6). As shown in Fig. 1A, MIA PaCa-2 cells exposed to EGCG for shorter period ( $8 \mathrm{~h}$ ) did not undergo significant apoptosis (PARP cleavage). At the same time period, EGCG also cannot activate caspase- 8 (Fig. 1A, lane 2). However remarkable activation of caspase-8 was noted following 24-h treatment of EGCG. Similar kinetics of activation was observed for caspase-9 (Fig. 1B).

Among the two major apoptotic pathways such as mitochondrial 'intrinsic' and transmembrane 'extrinsic', the latter one comprises of activation of death receptors (DR) such as Fas, TNF receptor 1 , DR4 or DR5 $(6,27)$. Apoptotic signal transduction cascade by extrinsic pathway ensues by the recruitment of DR associated molecules such as FADD to engage initiator caspase such as caspase- 8 . The activation of initiator caspases such as caspase- 8 can cleave a proapoptotic protein Bid and translocation of truncated Bid (t-Bid) to mitochondria can orchestrate mitochondrial events that can result in biochemical as well as morphological alterations implicated with programmed cell death. In EGCG exposed pancreatic cancer cells, we also observed Bid cleavage 
A.
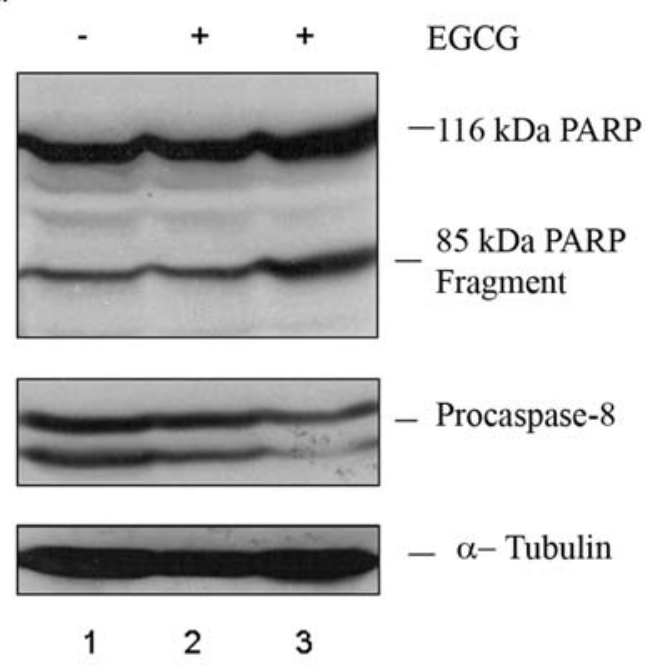

B.

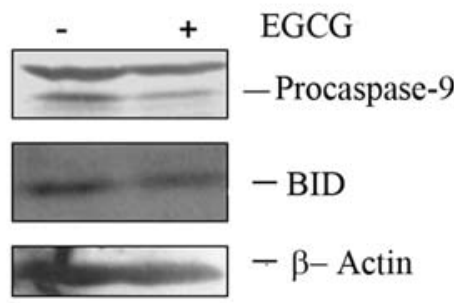

C.

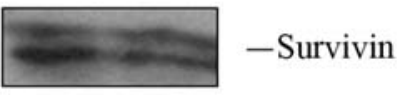

D.

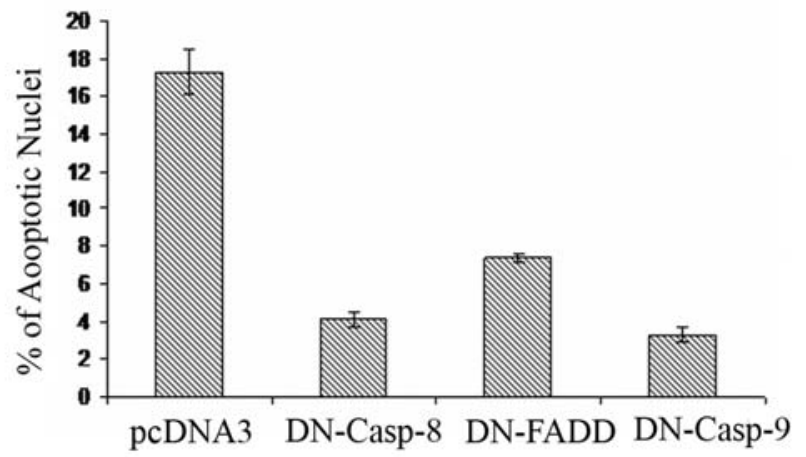

Figure 1. EGCG mediated death of pancreatic carcinoma cells requires both initiator and executioner caspases. A-C, MIA PaCa- 2 cells were exposed to $50 \mu \mathrm{g} / \mathrm{ml}$ EGCG for 8-24 h. Cell lysate was subjected to Western blot analysis with PARP (A), procaspase-8 (A), procaspase-9 (B), BID (B) and Survivin (C) antibodies . D, Determination of EGCG induced apoptosis following exogenous expression of dominant negative constructs of caspase-9, -8 and FADD. Cells were transfected with either vector (pcDNA3) or the indicated constructs by calcium phosphate co-precipitation method. Twenty-four hours post transfection, cell culture media was replaced with the fresh media containing $50 \mu \mathrm{g} / \mathrm{ml}$ EGCG. Following $24 \mathrm{~h}$ of EGCG treatment, cells were harvested and stained with DAPI for scoring apoptotic nuclei.

(Fig. 1B) which is most likely executed by activated caspase-8 Moreover, EGCG treatment can down-regulate Survivin (Fig. 1C), that is known to inhibit caspase activation therefore leading to negative regulation of apoptosis or programmed cell death.

To demonstrate whether FADD as well as caspase- 8 are required for EGCG mediated death of pancreatic cancer cells, we determined the extent of apoptosis in cells transfected with pcDNA3 vector, DN-caspase-8 and DN-FADD respectively. In contrast to empty vector, all these dominant negative constructs inhibited EGCG-induced apoptosis (Fig. 1D). We also investigated the effect of dominant negative construct of executioner caspase-9 on EGCG-induced cell death. The blocking of apoptosis by these DN constructs corroborates our biochemical data on activation of both caspase- 8 and -9 . Thus, EGCG-mediated demise of pancreatic cancer cells requires both caspase- 8 and -9 .

Up-regulation of death receptors in EGCG sensitive pancreatic cancer cells. The significant inhibition of EGCG-induced cell death by transfection of dominant negative construct of FADD (Fig. 1D) prompted us to investigate the involvement of the death receptors in this process. In general, death receptors mediate apoptosis by recruiting FADD to the oligomerized death receptor complex where FADD facilitates the binding and activation of procaspase- 8 or -10 . Accordingly, we performed RNase protection assay (RPA) using multiprobe template set. The multiprobe RPA systems comprise of a series of templates with distinct length and sequence of selective mRNA species. Here we used specific probes for genes encoding death receptor (DR) family members (DR3, DR4, DR5, Fas, and FasL), caspase-8. Sequences from GAPDH and L32 mRNA were used as internal controls. Quantitation of death related genes revealed by RPA analysis demonstrates 1.5-2-fold increase in of DR4, DR5 and Fas mRNA (Fig. 2A) levels in EGCG exposed MIA PaCa-2 cells. On the contrary, mRNA levels of DRs remain unaltered in EGCG challenged Hs766T cells (Fig. 2C). Of note, metastatic pancreatic cancer cells Hs766T are refractory to EGCGinduced apoptosis (4). In order to corroborate our RPA data, cellular extract of EGCG-exposed MIA PaCa-2 cells was subjected to immunoblot analysis. Western blot analyses (Fig. 2B) clearly demonstrate increased levels of members (Fas, DR5) of death receptor family in MIA PaCa-2 cells when exposed for $24 \mathrm{~h}$ to EGCG.

Synergistic action of EGCG and TRAIL on pancreatic carcinoma cell death. Our data indicating the ability of EGCG to up regulate death receptors in cultured pancreatic carcinoma cells led us to test whether EGCG in combination with death receptor ligand TRAIL can enhance the apoptosis inducing effect of EGCG. Tumor necrosis factor (TNF)-related 
A.

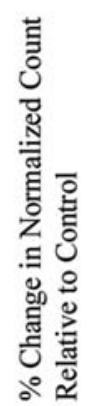

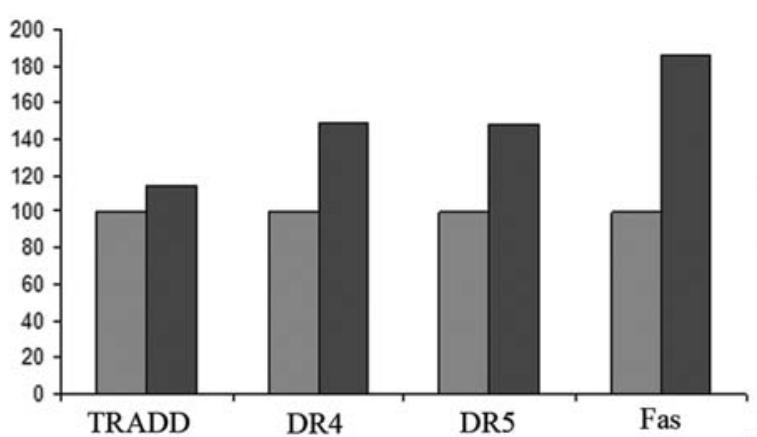

TRADD
DR4
DR5

Fas

\section{MIA PaCa-2 Cells}

C. Hs $766 \mathrm{~T}$ Cells

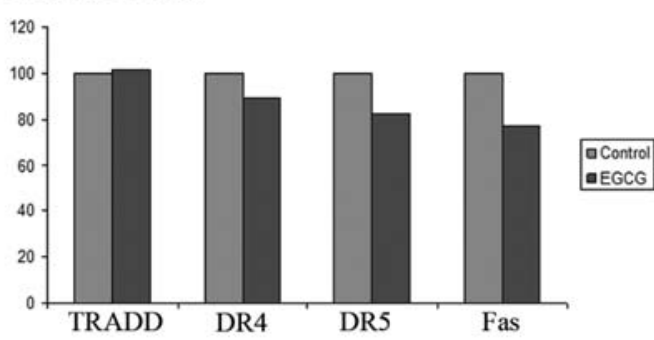

A.

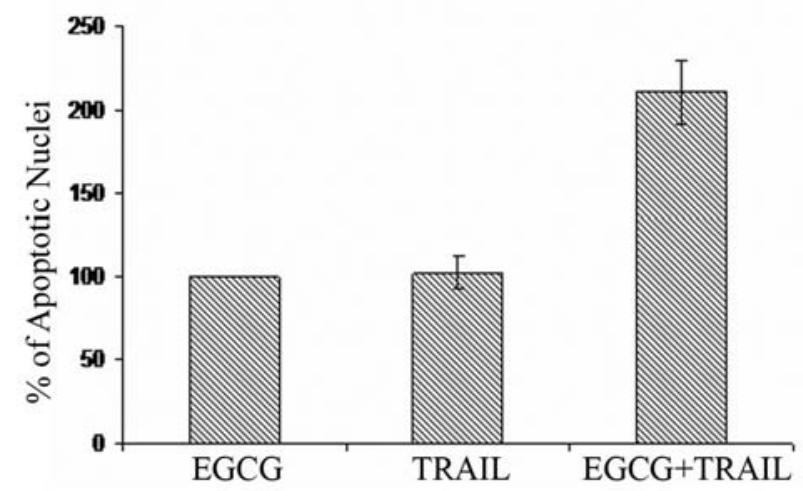

B.

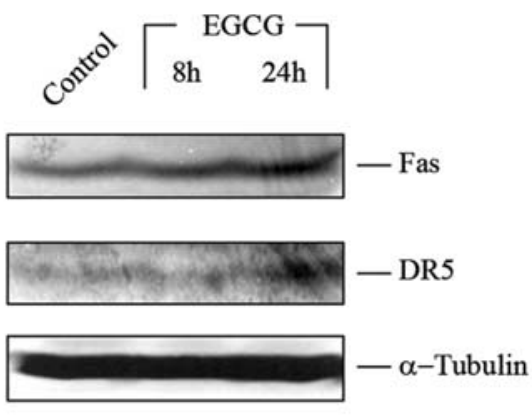

Figure 2. Effect of EGCG on death receptor related genes. A and C, Quantitation of death related genes revealed in RPA. MIA PaCa-2 (A) and Hs766T (C) cells were treated with or without EGCG for a period of $24 \mathrm{~h}$. Total RNA was used in ribonuclease protection assay (RPA) using hAPO-3d probe (BD Biosciences). ImageQuant software was used to scan protected bands and counts were normalized against house keeping gene L32. Percent change in normalized count relative to control is presented. B, Induction of death receptor proteins Fas and DR5 following EGCG exposure. Immunoblot analysis of total cellular proteins isolated from EGCG exposed MIA PaCa-2 cells against Fas and DR5 antibodies.

B.

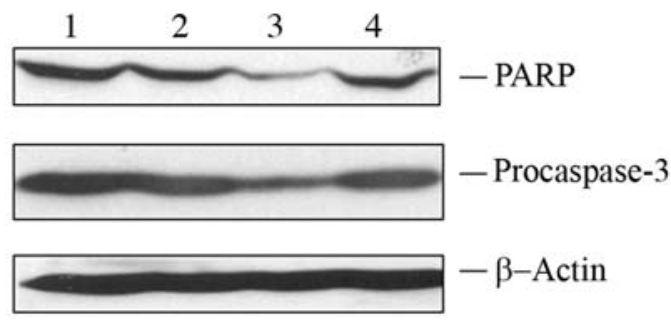

Figure 3. Combinatorial effect of EGCG and TRAIL on the induction of apoptosis in MIA PaCa-2 cells. MIA PaCa-2 cells were exposed to either $50 \mu \mathrm{g} / \mathrm{ml}$ EGCG or $5 \mathrm{ng} / \mathrm{ml}$ of recombinant human TRAIL or $50 \mu \mathrm{g} / \mathrm{ml}$ EGCG and $5 \mathrm{ng} / \mathrm{ml}$ TRAIL together for $16 \mathrm{~h}$. A, Cell death was determined by nuclear staining as described in Fig. 1. The extent of apoptosis in EGCG exposed cells was considered to be $100 \%$. B, Following designated treatment, cell free extract was subjected to Western blot analysis using monoclonal antibody against PARP and procaspase-3. Lane 1, control; lane 2, EGCG; lane 3, EGCG and TRAIL together; lane 4, TRAIL.

apoptosis inducing ligand (TRAIL) is a type II transmembrane cytokine and interacts with death receptors (DR4, DR5) to execute apoptosis in a variety of cancer cells $(6,27,28)$. As evident in Fig. 3A, TRAIL can further sensitize MIA PaCa-2 cells to EGCG-induced apoptosis ( $>2$-fold). We next sought to affirm the synergy between EGCG and TRAIL by analysis of biochemical markers such as PARP cleavage or caspase-3 activation. The combination treatment of EGCG and TRAIL was very effective for enhanced PARP cleavage as well as for the disappearance of procaspase-3 (Fig. 3B).
Combination treatment of EGCG and TRAIL diminishes survival of MIA PaCa-2 cells. As shown in Fig. 4, the potential therapeutic synergy by combining EGCG and TRAIL was noted by significant apoptosis of pancreatic carcinoma cells. Next, we were interested to examine whether the combination therapy would exert any effect on colony growth. As evident from clonogenic survival assay (Fig. 4), the number of colonies in EGCG or TRAIL treated MIAPaCa- 2 cells was much higher than those formed by combination treatment (Fig. 4, panel IV vs. panels II and III,). Our in vitro experiments 
A.
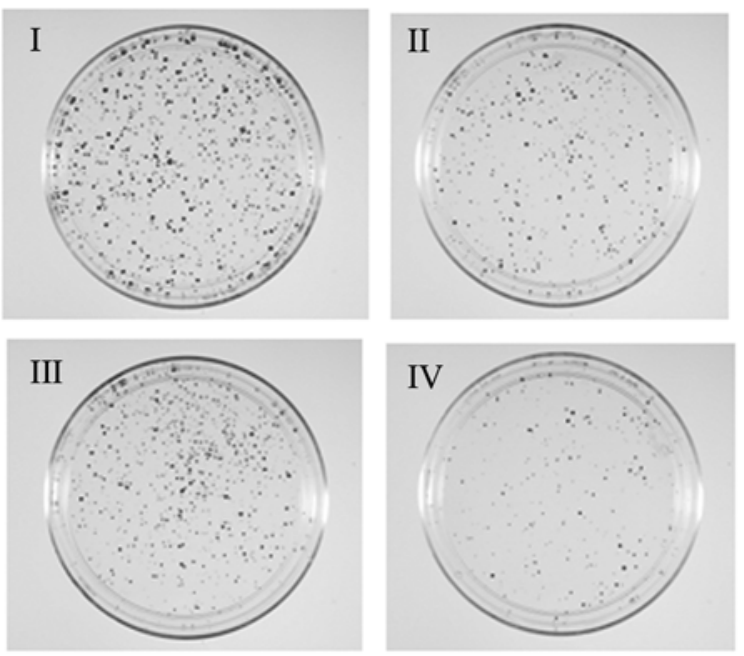

B.

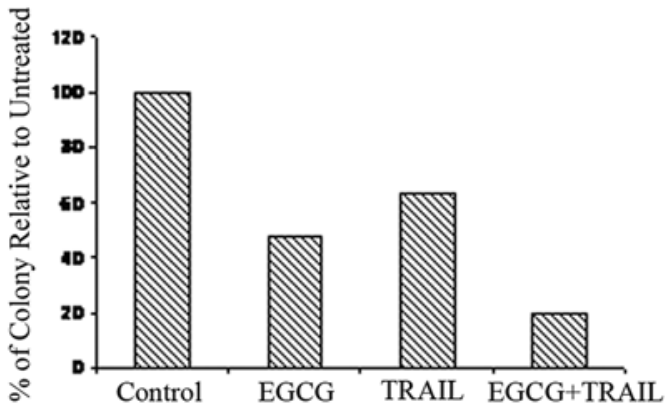

Figure 4. Decreased clonogenic survival of pancreatic carcinoma cells due to combinatorial treatment of EGCG and TRAIL. A, Clonogenic cell survival assay was employed to determine the long-term survival ability of MIA PaCa-2 cells in response to several treatment regimens as described in Fig. 3. Panel I, control; panel II, EGCG; panel III, TRAIL; panel IV, EGCG and TRAIL together. B, Manual count of colonies was presented. Note that extent of colony formation in untreated control was considered to be $100 \%$.

demonstrate that the combination of EGCG and TRAIL resulted in enhanced growth inhibition compared with EGCG or TRAIL alone.

\section{Discussion}

Cancer of the pancreas stands out as a highly lethal disease with the poorest likelihood of survival among all of the major malignancies. Pancreatic cancer is often far advanced by the time symptoms occur and the diagnosis is established. As indicated by 8 -year survival rates of $<5 \%$, the successful treatment of this cancer is rare. The development of malignancies is a multi-step process that comprise of initiation, progression and finally establishment of metastasis (1-3). The multiple genetic alterations at each stage impart selective advantage over normal counterpart and might contribute to resistance to therapy of this malignancy. Targeting apoptotic signaling machinery in pancreatic cancer might be a useful approach from therapeutic/prevention standpoint.

The data presented in this report indicate that polyphenolic constituent of green tea can render pancreatic cancer cells sensitive to death receptor ligand TRAIL mediated apoptosis possibly by upregulation of members of the death receptor family. At present the molecular mechanism of EGCGinduced upregulation of cell surface Fas/DR4/DR5 is not clear. However, as observed earlier $(29,30)$ one possibility might be AP-1 mediated transcriptional regulation of death receptors. Our previous report implicated a link between JNK pathway and EGCG mediated cell death (4). Activated JNK might lead to increase AP-1 expression $(31,32)$. Interestingly, Fas promoter region has been shown to contain AP-1 binding site $(29,30)$.

Further studies with dominant negative constructs of FADD, caspase- 8 and -9 contribute to identify molecular components associated with EGCG mediated cell death of pancreatic carcinoma. The activation of death receptors signals the activation of caspase- 8 leading to cleavage of proapoptotic protein Bid (Fig. 1). The interaction of truncated Bid with Bax, either in the cytosol or on the mitochondrial surface, renders conformational changes in Bax. Proapoptotic Bax then inserts itself into outer mitochondrial membrane where it oligomerizes and facilitates release of cytochrome c from the intermembrane mitochondrial space. We have previously documented EGCG-induced Bax oligomerization and cytochrome c release from mitochondria to cytosol in MIA PaCa-2 cells (4). Here we have shown the disappearance of intact Bid in EGCG treated cells (Fig. 1B). In addition, the level of Survivin, which is known to be the potent endogenous inhibitor of processed caspase-3, -7 and -9 , is diminished following EGCG exposure (Fig. 1C). Our cumulative studies demonstrate the convergence of mitochondrial machinery of apoptosis with death receptor pathway in EGCG mediated apoptosis of pancreatic cancer cells. Thus, cross-talk between extrinsic and intrinsic pathway might play a pivotal role in initiating apoptotic signaling cascade by EGCG.

Based on this, we employed combination treatment of EGCG and TRAIL that diminished survival of pancreatic cancer cells MIA PaCa-2. As shown in Fig. 3, the potential therapeutic synergy by combining EGCG and TRAIL was noted by significant apoptosis of MIA $\mathrm{PaCa}-2$ pancreatic carcinoma cells. In parallel, clonogenic survival assay demonstrated enhanced growth inhibition of MIA PaCa-2 cells when treated with EGCG and TRAIL together (Fig. 4).

To the best of our knowledge, we report for the first time the efficacy of combination treatment of EGCG and TRAIL to sensitize demise of pancreatic cancer cells. In general, the constitutive activation of nuclear factor-kappaB is thought to contribute to develop TRAIL resistance in human pancreatic cancer cells (33). Thus the ability of EGCG to augment sensitivity of TRAIL bears translational potential for therapy of TRAIL resistant pancreatic cancer. 


\section{Acknowledgments}

This work was supported by NIH Grants CA 109181 and CA 137476. We also thank K. Haas for technical assistance.

\section{References}

1. Giovannetti E, Mey V, Nannizzi S, Pasqualetti G, Del Tacca M and Danesi R: Pharmacogenetics of anticancer drug sensitivity in pancreatic cancer. Mol Cancer Ther 5: 1387-1395, 2006.

2. Maitra A, Kern SE and Hruban RH: Molecular pathogenesis of pancreatic cancer. Best Pract Res Clin Gastroenterol 20: 211-226, 2006

3. Hruban RH, Adsay NV, Albores-Saavedra J, et al: Pathology of genetically engineered mouse models of pancreatic exocrine cancer: consensus report and recommendations. Cancer Res 66: 95-106, 2006.

4. Qanungo S, Das M, Haldar S and Basu A: Epigallocatechin-3gallate induces mitochondrial membrane depolarization and caspase-dependent apoptosis in pancreatic cancer cells. Carcinogenesis 26: 958-967, 2005.

5. Ju J, Lu G, Lambert JD and Yang CS: Inhibition of carcinogenesis by tea constituents. Semin Cancer Biol 17: 395-402, 2007.

6. Basu A, Castle VP, Bouzianne M, Bhalla KN and Haldar S: Cross talk between extrinsic and intrinsic cell death pathways in pancreatic cancer: synergistic action of estrogen metabolite and ligands of death receptor family. Cancer Res 66: 4309-4318, 2006.

7. Basu A and Haldar S: Dietary isothiocyanate mediated apoptosis of human cancer cells is associated with Bcl-xL phosphorylation. Int J Oncol 33: 657-663, 2008.

8. Basu A and Haldar S: 2-Methoxyestradiol mediated signaling network in pancreatic cancer. Front Biosci (In press).

9. Hecht SS: Chemoprevention of cancer by isothiocyanates, modifiers of carcinogen metabolism. J Nutr 129: S768-S774, 1999.

10. Howell A, Sims AH, Ong KR, Harvie MN, Evans DG and Clarke RB: Mechanisms of disease: prediction and prevention of breast cancer-cellular and molecular interactions. Nat Clin Pract Oncol 2: 635-646, 2005.

11. Stoner GD, Wang LS and Chen T: Chemoprevention of esophageal squamous cell carcinoma. Toxicol Appl Pharmacol 224: 337-349, 2007.

12. Siddiqui IA, Malik A, Adhami VM, Asim M, Hafeez BB, Sarfaraz S and Mukhtar H: Green tea polyphenol EGCG sensitizes human prostate carcinoma LNCaP cells to TRAIL-mediated apoptosis and synergistically inhibits biomarkers associated with angiogenesis and metastasis. Oncogene 27: 2055-2063, 2008.

13. Canene-Adams K, Lindshield BL, Wang S, Jeffery EH, Clinton SK and Erdman JW Jr: Combinations of tomato and broccoli enhance antitumor activity in dunning r3327-h prostate adenocarcinomas. Cancer Res 67: 836-843, 2007.

14. Shukla $S$ and Gupta S: Dietary agents in the chemoprevention of prostate cancer. Nutr Cancer 53: 18-32, 2005

15. Katiyar SK, Mohan RR, Agarwal R and Mukhtar H: Protection against induction of mouse skin pappilomas with low and high risk of conversion to malignancy by green tea polyphenols. Carcinogenesis 18: 497-502, 1997.
16. Gupta S, Hastak K, Ahmad N, Lewin JS and Mukhtar H: Inhibition of prostate carcinogenesis in TRAMP mice by oral infusion of green tea polyphenols. Proc Natl Acad Sci USA 98: 10350-10355, 2001.

17. Sartippour MR, Shao Z-M, Heber D, et al: Green tea inhibits vascular endothelial growth factor (VEGF) induction in breast cancer cells. J Nutr 132: 2307-2311, 2002.

18. Cao Y and Cao R: Angiogenesis inhibited by drinking tea. Nature 398: 381, 1999

19. Haldar S, Basu A and Croce CM: Serine-70 is one of the critical sites for drug-induced Bcl2 phosphorylation in cancer cells. Cancer Res 58: 1609-1615, 1998.

20. Basu A and Haldar S: Microtubule-damaging drugs triggered Bcl2 phosphorylation requirement of phosphorylation on both serine-70 and serine- 87 residues of $\mathrm{Bcl} 2$ protein. Int $\mathrm{J}$ Oncol 13: 659-664, 1998.

21. Basu A, Das M, Qanungo S, Fan X-U, Dubois G and Haldar S: Proteasomal degradation of human peptidyl prolyl isomerase Pin1-pointing phospho Bcl2 toward dephosphorylation. Neoplasia 4: 218-227, 2002.

22. Haldar $\mathrm{S}$, Jena $\mathrm{N}$ and Croce $\mathrm{CM}$ : Inactivation of $\mathrm{Bcl} 2$ by phosphorylation. Proc Natl Acad Sci USA 92: 4507-4511, 1995.

23. Basu A and Haldar S: Identification of a novel Bcl-xL phosphorylation site regulating the sensitivity of Taxol or 2-methoxyestradiol induced apoptosis. FEBS Lett 538: 41-47, 2003.

24. Qanungo S, Haldar S and Basu A: Restoration of silenced Peutz-Jeghers syndrome gene, LKB 1, induces apoptosis in pancreatic carcinoma cells. Neoplasia 5: 367-374, 2003.

25. Qanungo S, Basu A, Das M and Haldar S: 2-Methoxyestradiol induces mitochondria-dependent apoptotic signaling in pancreatic cancer cells. Oncogene 21: 4149-4157, 2002.

26. Munshi A, Hobbs M and Meyn RE: Clonogenic cell survival assay. Methods Mol Med 110: 21-28, 2005.

27. Ashkenazi A and Dixit VM: Death receptors: signaling and modulation. Science 281: 1305-1308, 1998.

28. Guo F, Sigua C, Tao J, et al: Cotreatment with histone deacetylase inhibitor LAQ824 enhances Apo-2L/tumor necrosis factorrelated apoptosis inducing ligand-induced death inducing signaling complex activity and apoptosis of human acute leukemia cells. Cancer Res 64: 2580-2589, 2004.

29. Li XR, Chong A, Wu J, et al: Transcriptional regulation of Fas gene expression by GA-binding protein and AP-1 in T cell antigen receptor CD3 complex-stimulated T cells. J Biol Chem 274: 35203-35210, 1999.

30. Guan B, Yue P, Lotan R and Sun S-Y: Evidence that the death receptor 4 is regulated by activator protein 1 . Oncogene 21 : 3121-3129, 2002.

31. Zou W, Liu X, Yue P, et al: c-Jun NH2-terminal kinase-mediated upregulation of death receptor 5 contributes to induction of apoptosis by the novel synthetic triterpenoid methyl-2-cyano3,12-dioxooleana-1, 9-dien-28-oate in human lung cancer cells. Cancer Res 64: 7570-7578, 2004.

32. Xia S, Rosen EM and Laterra J: Sensitization of glioma cells to fas-dependent apoptosis by chemotherapy-induced oxidative stress. Cancer Res 65: 5248-5255, 2005.

33. Khanbolooki S, Nawrocki ST, Arumugam T, et al: Nuclear factor-kappaB maintains TRAIL resistance to human pancreatic cancer cells. Mol Cancer Ther 5: 2251-2260, 2006. 